\title{
Comparative Experimental İnvestigation and Application of Five Classic Pre-Trained Deep Convolutional Neural Networks via Transfer Learning for Diagnosis of Breast Cancer
}

\author{
Hidir Selcuk Nogay ${ }^{1}$, Tahir Cetin Akinci ${ }^{2 *}$, Musa Yilmaz ${ }^{3}$ \\ 1 Department of Electrical and Energy, Kayseri University, Kayseri, Turkey \\ 2 Department of Electrical Engineering, Istanbul Technical University, Istanbul, Turkey \\ 3 Department of Electrical and Electronics Eng., Batman University, Batman, Turkey \\ * Corresponding author's email: akincitc@itu.edu.tr
}

\begin{abstract}
In this study, for the diagnosis and classification of breast cancer, the authors used and applied five classical pretrained deep convolutional neural network models (DCNN) which have proven successful many times in different fields (ResNet-18, AlexNet, GoogleNet and SuffleNet). In order to make pre-trained DCNN models suitable for the purpose of this study, some layers were updated according to the new situation by using the transfer learning technique. The weights of all layers used in these five pre-trained DCNN models were not changed. Instead, new weights were given to the new layers so that new layers adapt faster to the emerging new DCNN models. With these five pre-trained DCNN models, a quadruple classification as "cancer", "normal", "actionable" and "benign", and a binary classification as "actionable + cancer" and "normal + benign" was realized. With these two separate classification and diagnosis studies, comparative experimental examination and analysis of pre-trained DCNN models for breast cancer diagnosis were carried out. In the study, it was concluded that successful results can be achieved with pre-trained DCNN models without extra time-consuming procedures such as feature extraction, as well as DCNN can perform quite successfully in cancer diagnosis and image comment.
\end{abstract}

Keywords: breast cancer, classification, deep learning, DCNN, transfer learning, diagnosis.

\section{INTRODUCTION}

Breast cancer is the most common cancer among women, and this type of cancer is also recognized as one of the major causes of death (Cuzick et al., 2020; Lei et al., 2021) (Patnaik, Byers, DiGuiseppi, Dabelea, \& Denberg, 2011) (Oh et al., 2020). The occurrence of breast cancer is related to many factors (Yousefi et al., 2020). This cancer can be seen in different regions of the world with different effects and rates. Breast cancer is highly influenced by such factors as population structure, genetic predisposition, lifestyle, physical activity, smoking and alcohol use, dietary habits, and environment. This diversity also affects mortality rates. Statistics show that breast cancer cases are on an increasing trend. Although health screenings and early diagnosis are considered an advantage in the treatment process, the side effects and high cost of the treatments are its disadvantages.

The incidence for non-sick individuals at risk to develop breast cancer shows that breast cancer is the second most common cancer in the world and is the most common cancer among women.

The diagnosis of breast cancer is made via biopsy by collecting tissue samples from the breast area by pathologists. Afterwards, these tissues are examined with a microscope, and the histopathology images obtained from here are completed by the pathologists classifying them as cancerous or non-cancerous images. Therefore, accurate and reliable image interpretation emerges as 
an important need in cancer diagnosis. Machine learning and deep learning methods based on image data show promise in the interpretation of medical images. There are many studies in the literature regarding the computer-aided diagnosis of breast cancer. Kowal et al. (Kowal, Filipczuk, Obuchowicz, Korbicz, \& Monczak, 2013) made automatic classification of images related to breast cancer. The study was planned in two stages, in the first stage, the foreground and background segmentation in the images with the adaptive threshold method, and in the second stage, the nucleus, red blood cells, and other features are distinguished. In the classification, $500 \mathrm{im}$ ages were studied using three classifiers, Bayes, $\mathrm{k}$-nearest neighbor (K-NN ), and decision trees (DT). In the approach proposed by Berbar (Berbar, 2018), the wavelet-based contourlet method was used to classify the mammography images. In addition, entropy, energy, inverse difference method, and features have been extracted by basic statistical analysis. Many studies on breast cancer focus on the processing of images based on Artificial Neural Networks (ANN) (Hakkoum, Idri, \& Abnane, 2021) (Rawal, Rawal, Shah, \& Patel, 2020) (Gupta, Vijay, Pahadiya, \& Applications, 2020). Again, the SVM method has been widely used in the literature for the classification of the breast cancer data (Singh \& Kumar, 2020) (Liu et al., 2020) (Vrigazova, 2020). Some of the widely used methods for classifying the breast cancer images are the signal processing methods (Rasheed, Younis, Qadir, \& Bilal, 2021) (Zhang \& Li, 2020) (de Santana, Pereira, da Silva, \& dos Santos, 2021) (Bharti, Sahoo, Shukla, \& Pradhan, 2020). Recently, the machine learning (ML) methods, which is used for classification and analysis, has begun to be widely used in breast cancer diagnosis (Vaka, Soni, \& Reddy, 2020) (Binder et al., 2021) (Hou et al., 2020).

The CNN methods used in the classification of images in breast cancer diagnosis give very successful results. The R-CNN, 3D - CNN, and MA-CNN methods have been used in recent years, (Agnes, Anitha, Pandian, \& Peter, 2020) (Lei et al., 2021) (Desai \& Shah, 2020).

The cancer diagnosis and classification studies mentioned above, with the exception of $\mathrm{CNN}$, all require a feature extraction process. In the DCNN method, feature extraction is performed automatically. In studies conducted by designing a CNN model from scratch, the number of data should be quite high and reliable. Therefore, applying the classical DCNN models, which have been designed and proven successful before, by using the transfer learning approach can be more effective, faster, and more convincing.

In this study, 5 classical DCNN models (Alexnet, Googlenet, Resnet18, Squeezenet, and Shufflenet) were used for breast cancer diagnosis and classification, using the transfer learning approach. In the study, quadruple classification as cancerous, normal, actionable and benign, as well as a dual classification and diagnostic study as 'actionable + cancer' and 'normal + benign' were carried out. For this purpose, five different DCNN models have been implemented by changing and redesigning some layers, owing to the transfer learning approach. The motivation of the study is to prove that classical deep learning methods can be used in the diagnosis and classification of breast cancer by using the transfer learning approach and to shed light on the future studies in this field.

\section{Obtaining and editing the data set}

The data used in this study were obtained from the database published on the website of The Cancer Imaging Archive (TCIA) (Clark et al. 2013). Detailed information about the data and database can be found at https://www.cancerimagingarchive.net/collections/. The dataset consists of four categories of normal, actionable, biopsy-proven benign, and biopsy-proven cancer cases, and digital breast tomosynthesis images (Buda et al.2020). Two separate data sets were used for two separate classifications in the study. A total of 3592 computerized tomography (CT) images of 985 participants were obtained from the database. However, for the purpose of the study, only a limited part of these image data was selected as shown in Table 1 and used by cropping and reducing the pixels. Table 1 presents the summaries of the data sets used in this study.

\section{Architecture of the DCNN models}

The classical DCNN models (Alexnet, Resnet18, Googlenet, Squeezenet, and Shufflenet) (Szegedy et al. 2015; He et al. 2016; Abhinav 2018; Krizhevsky, et al. 2017; Zhang et al. 2018) were trained from scratch with a new dataset owing to the transfer learning method. The design, training, and testing processes of the DCNN models were carried out in MATLAB environment, 
Table 1. Summary of the data sets

\begin{tabular}{|c|c|c|c|c|}
\hline Datasets & $\begin{array}{l}\text { Class } \\
\text { Number }\end{array}$ & Groups & Size & $\begin{array}{l}\text { Total } \\
\text { Size }\end{array}$ \\
\hline \multirow{4}{*}{ Data1 } & 1 & Cancer & 50 & \multirow{4}{*}{200} \\
\hline & 2 & Benign & 50 & \\
\hline & 3 & Actionable & 50 & \\
\hline & 4 & Normal & 50 & \\
\hline \multirow{2}{*}{ Data2 } & 1 & Cancer+Actionable (CA) & 100 & \\
\hline & 2 & Normal+Benign (NB) & 100 & \\
\hline
\end{tabular}

and the computer used was equipped with NVIDIA GeForce 940MX, 6040 MB GPU.

\section{Convolution Layer}

In the convolution layer, the input matrix " $\mathrm{A}$ " is filtered with a smaller filter matrix of size $n_{k}$ $\times n_{k}$ (kernel). Stride (s) is the step of shifting the filter matrix. " $\mathrm{B}$ " is the output matrix obtained as a result of the convolution (Michelucci 2019).

$B_{i j}=(A * K)_{i j}=\sum_{f=0}^{n_{K}-1} \sum_{h=0}^{n_{K}-1} A_{i+f, j+h} K_{i+f, j+h}(1)$

where: $K$ is the filter matrix, $A$ is the input, and $B$ is the output matrix. The "padding" (p) number is the coefficient used to bring the smaller matrix to its original size after filtering. Taking into account the number $\mathrm{P}$, the output matrix B can be expressed as follows (Michelucci 2019).

$$
n_{B}=\left\lfloor\frac{n_{A}+2 p-n_{K}}{s}+1\right\rfloor
$$

\section{Classification and Softmax Layer}

The classification layer is the last layer connected after the softmax layer in a DCNN architecture, and the cross-entropy loss is calculated in this layer.

$$
y_{r}(x)=\frac{\exp \left(a_{r}(x)\right)}{\sum_{j=1}^{k} \exp \left(a_{j}(x)\right)}
$$

The softmax function produces outputs between $[0,1]$ that show the probability of each given input belonging to a class (Bishop 2006). In Equation 3, $a_{r}$ indicates the conditional probability of the sample belonging to the class $r$.

\section{Transfer Learning}

"Transfer learning" is a technique that allows retraining and using a trained DCNN

\begin{tabular}{|c|c|c|c|c|c|}
\hline DCNN Models & Alexnet & Googlenet & Squeezenet & Resnet18 & Shufflenet \\
\hline Input Image Size & $227 \times 227$ & $224 \times 224$ & $227 \times 227$ & $224 \times 224$ & $224 \times 224$ \\
\hline $\begin{array}{l}\text { Number of } \\
\text { Convolution } \\
\text { Layer }\end{array}$ & 5 & 57 & 26 & 20 & 49 \\
\hline Number of Pooling & 3 & 14 & 4 & 2 & 4 \\
\hline $\begin{array}{l}\text { Number of Fully } \\
\text { Connected }\end{array}$ & 3 & 1 & - & 1 & 1 \\
\hline $\begin{array}{l}\text { Number of } \\
\text { Dropouts }\end{array}$ & 2 & 1 & 1 & - & - \\
\hline $\begin{array}{l}\text { Number of } \\
\text { Normalization }\end{array}$ & 2 & 2 & - & 20 & 49 \\
\hline $\begin{array}{l}\text { Number Of } \\
\text { Activation Function } \\
\text { (ReLu) }\end{array}$ & 7 & 57 & 26 & 17 & 33 \\
\hline $\begin{array}{l}\text { Total Number of All } \\
\text { Layers }\end{array}$ & 25 & 144 & 68 & 72 & 136 \\
\hline $\begin{array}{l}\text { Rearranged } \\
\text { Layers via } \\
\text { Transfer Learning }\end{array}$ & $\begin{array}{l}\text { Classification, } \\
\text { Softmax and Last } \\
\text { Fully Connected }\end{array}$ & $\begin{array}{l}\text { Classification, } \\
\text { Softmax and Last } \\
\text { Fully Connected }\end{array}$ & $\begin{array}{l}\text { Classification, } \\
\text { and Last } \\
\text { Convolution }\end{array}$ & $\begin{array}{l}\text { Classification, } \\
\text { Softmax and Last } \\
\text { Fully Connected }\end{array}$ & $\begin{array}{l}\text { Classification, } \\
\text { Softmax and Last } \\
\text { Fully Connected }\end{array}$ \\
\hline
\end{tabular}

Table 2. Structural information for all DCNN models 
model for another task. In order to retrain and test the DCNN models with the transfer learning method, some layers have been removed and replaced with new layers to perform binary classification (Cancer + Actionable and Benign + Normal) and quadruple classification (Cancer, Actionable, Benign, and Normal). Table 2 shows the arrangements made for each model.

In each DCNN, each convolution layer is connected to the rectified linear unit (ReLU) activation function. In the study, the learning rate of the new layers used in DCNN models was slightly increased in order for them to adapt to new models quickly and to achieve rapid learning. The learning rate of the old layers was not changed.

\section{Training and testing of the DCNN models}

The study was tested with a fivefold cross-validation technique and shows the highest test result obtained, because all test results were very close to each other. The hyper parameters for training and testing are shown in Table 3. Training and testing continue until the loss reaches its minimum value.

$$
\text { Loss }=-\sum_{i=1}^{N} \sum_{j=1}^{K} t_{i j} \ln y_{i j}
$$

Table 3. The hyperparameters of the DCNN models

\begin{tabular}{|l|c|}
\hline Maximum epoch & 30 \\
\hline Maximum iteration & 90 \\
\hline Iteration per epoch & 3 \\
\hline Validation Frequency & 5 Iteration \\
\hline Initial learning rate & 0.001 \\
\hline Mini batch size & 50 \\
\hline Learning rate schedule & Constant \\
\hline
\end{tabular}

In the loss equation (4), $N$ is the number of samples, $K$ is the number of classes and $t_{i j}$ is the sample $i$ in the $j$ class, and $y_{i j}$ is the output for sample $j$ class $i$ (Bishop 2006).

\section{RESULTS}

The main purpose of this study was to form and test the DCNN models for diagnosis of breast cancer. In the time allocated for this study, a total of five DCNN models were applied. The results obtained in this study are shown in Table 4. As a result of training and testing with Data 1 , the quadruple classification (cancer, benign, normal and actionable) was realized. It is understood from Table 4 that the highest accuracy rate was achieved with Alexnet and Squeezenet as a result of the test performed with Data 1 . The accuracy rates range from 66 to $76 \%$ in test results with Data 1. From Table 4, when the binary classification (cancer + actionable, benign+normal) results with Data 2 are examined, it can be said that Resnet-18 has reached the highest accuracy rate and Squeezenet has reached the lowest accuracy rate.

The same results can be observed from the confusion matrices in Table 5 and 6. The confusion matrix obtained with Data 1 is shown in Table 5, including both models. It is seen that success has been achieved with all methods in "Actionable" and "Normal" predictions in quadruple classification.

The confusion matrices obtained for Data 2 is given in Table 6 According to the confusion matrix obtained with Data 2, the model that the most correctly predicts the goal is Resnet- 18 .

Accuracy and loss curves obtained with Data 1 are given in Figure 1 and Figure 2 respectively.

Table 4. Accuracy and loss results with all the DCNN models

\begin{tabular}{|c|c|c|c|c|c|c|c|}
\hline \multirow[b]{2}{*}{ DCNN Models } & \multicolumn{2}{|c|}{ Training } & \multicolumn{3}{|c|}{ Testing } & \multirow{2}{*}{$\begin{array}{l}\text { Time Elapsed } \\
\text { (hh:mm:ss) }\end{array}$} & \multirow[b]{2}{*}{ Data Sets } \\
\hline & $\begin{array}{c}\text { Accuracy } \\
(\%)\end{array}$ & Loss & $\begin{array}{c}\text { Accuracy } \\
(\%)\end{array}$ & Loss & $\begin{array}{l}\text { Num. } \\
\text { Corrects }\end{array}$ & & \\
\hline Alexnet & 100.00 & 0.0150 & 75.00 & 1.2173 & 36 & 00:00:31 & \multirow{5}{*}{ Data 1} \\
\hline Googlenet & 100.00 & 0.0202 & 68.75 & 1.0380 & 33 & 00:01:19 & \\
\hline Resnet18 & 100.00 & 0.0072 & 72.92 & 0.8614 & 35 & 00:00:54 & \\
\hline Squeezenet & 100.00 & 0.0142 & 75.00 & 1.0408 & 36 & $00: 00: 45$ & \\
\hline Shufflenet & 100.00 & 0.0066 & 64.58 & 0.7668 & 32 & 00:01:11 & \\
\hline Alexnet & 100.00 & 0.0235 & 82.00 & 0.9551 & 41 & $00: 00: 24$ & \multirow{4}{*}{$\begin{array}{l}\text { Data } 2 \\
\text { (Binary) }\end{array}$} \\
\hline Googlenet & 100.00 & 0.0362 & 78.00 & 9.4595 & 39 & 00:01:23 & \\
\hline Resnet18 & 100.00 & 0.0041 & 86.00 & 0.3933 & 43 & 00:00:54 & \\
\hline Squeezenet & 98.00 & 0.0498 & 66.00 & 1.0415 & 33 & 00:01:04 & \\
\hline
\end{tabular}


Table 5. Confusion matrixes for Data 1 (quad. classification)

\begin{tabular}{|c|c|c|c|c|c|c|}
\hline \multirow{3}{*}{ Models } & \multicolumn{6}{|c|}{ Confusion matrix for Data 1} \\
\hline & & \multicolumn{4}{|c|}{ Predicted class } \\
\hline & & & Actionable & Benign & Cancer & Nromal \\
\hline Alexnet & \multirow{20}{*}{ True class } & \multirow{5}{*}{ Actionable } & 9 & 2 & 1 & \\
\hline Googlenet & & & 11 & & 1 & \\
\hline Resnet-18 & & & 11 & 1 & & \\
\hline Squeezenet & & & 11 & & 1 & \\
\hline Shufflenet & & & 11 & 1 & & \\
\hline Alexnet & & \multirow{5}{*}{ Benign } & - & 11 & 1 & \\
\hline Googlenet & & & 1 & 4 & 5 & 2 \\
\hline Resnet-18 & & & 2 & 6 & 4 & \\
\hline Squeezenet & & & & 6 & 5 & 1 \\
\hline Shufflenet & & & 1 & 8 & 2 & 1 \\
\hline Alexnet & & \multirow{5}{*}{ Cancer } & 3 & 3 & 6 & \\
\hline Googlenet & & & 3 & 2 & 6 & 1 \\
\hline Resnet-18 & & & 2 & 4 & 6 & \\
\hline Squeezenet & & & 3 & 2 & 7 & \\
\hline Shufflenet & & & 4 & 4 & 3 & 1 \\
\hline Alexnet & & \multirow{5}{*}{ Normal } & & 1 & 1 & 10 \\
\hline Googlenet & & & & & & 12 \\
\hline Resnet-18 & & & & & & 12 \\
\hline Squeezenet & & & & & & 12 \\
\hline Shufflenet & & & & & 2 & 10 \\
\hline
\end{tabular}

Table 6. Confusion matrixes for Data 2. AC:Actionable+Cancer; BN: Benign+Normal

\begin{tabular}{|c|c|c|c|c|}
\hline \multirow{2}{*}{ Models } & & & \multicolumn{2}{|c|}{ Predicted class } \\
\hline & & & $A C$ & $\mathrm{BN}$ \\
\hline Alexnet & \multirow{10}{*}{ True class } & \multirow{5}{*}{$A C$} & 17 & 8 \\
\hline Googlenet & & & 16 & 9 \\
\hline Resnet-18 & & & 23 & 2 \\
\hline Squeezenet & & & 16 & 9 \\
\hline Shufflenet & & & 17 & 8 \\
\hline Alexnet & & \multirow{5}{*}{$\mathrm{BN}$} & 1 & 24 \\
\hline Googlenet & & & 2 & 23 \\
\hline Resnet-18 & & & 5 & 20 \\
\hline Squeezenet & & & 8 & 17 \\
\hline Shufflenet & & & 7 & 18 \\
\hline
\end{tabular}

It is possible to understand which method is more successful from these curves and make comparisons with each other. In Figure 1, it is clear that all methods achieve very close results and their accuracy rates are between 66-76\%. In Figure 2, in terms of loss, it can be said that Shufflenet is closer to zero and more successful.
Test (validation) graphics can be used to see the results obtained from the study in a more concrete way. From figure 3, as a result of the binary classification made with Data 2, the process can be observed which method approximates most to $100 \%$. As can be seen from the graphs shown in Figure 3, with $86 \%$ of accuracy rate of Resnet- 18 

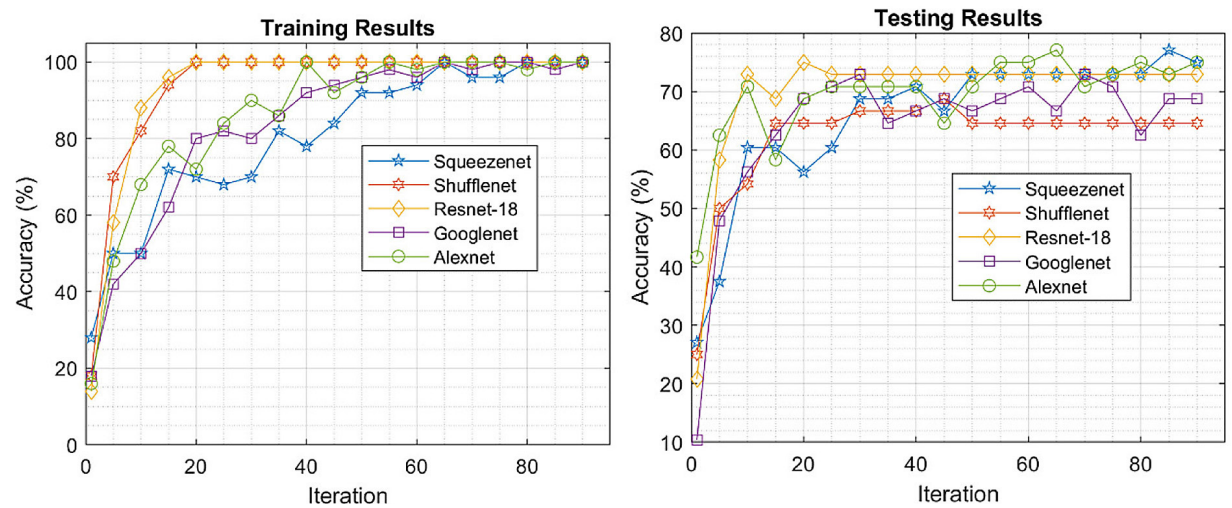

Fig. 1. Quadruple classification accuracy results with Data 1
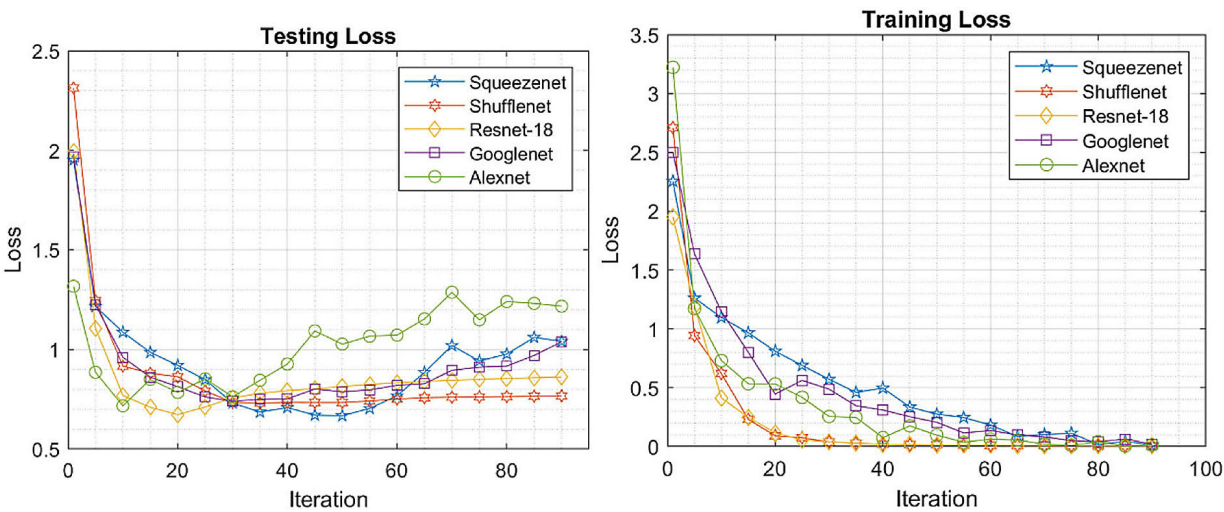

Fig. 2. Quadruple classification loss curves with Data 1
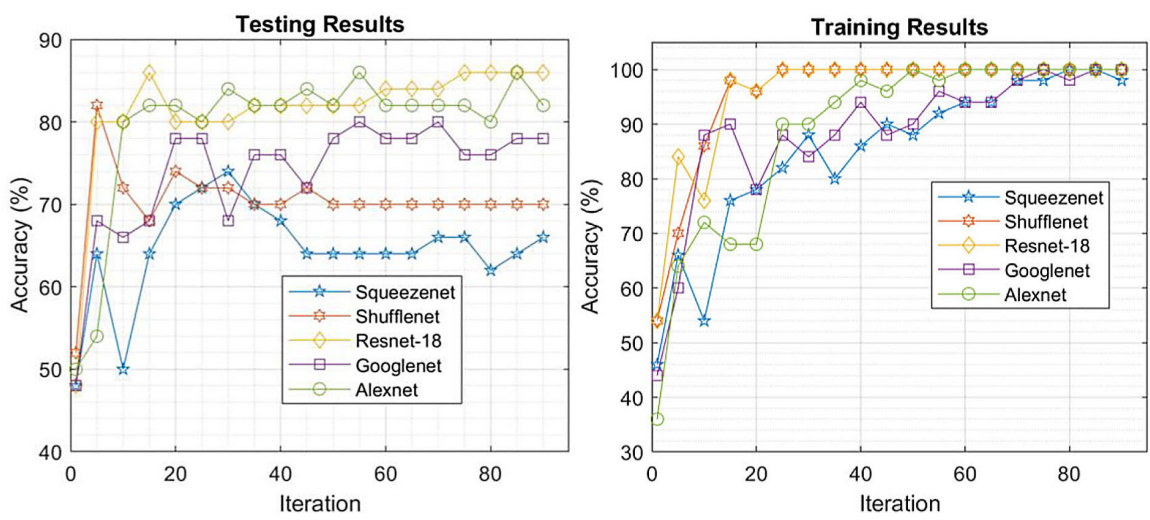

Fig. 3. Binary classification accuracy results with Data 2
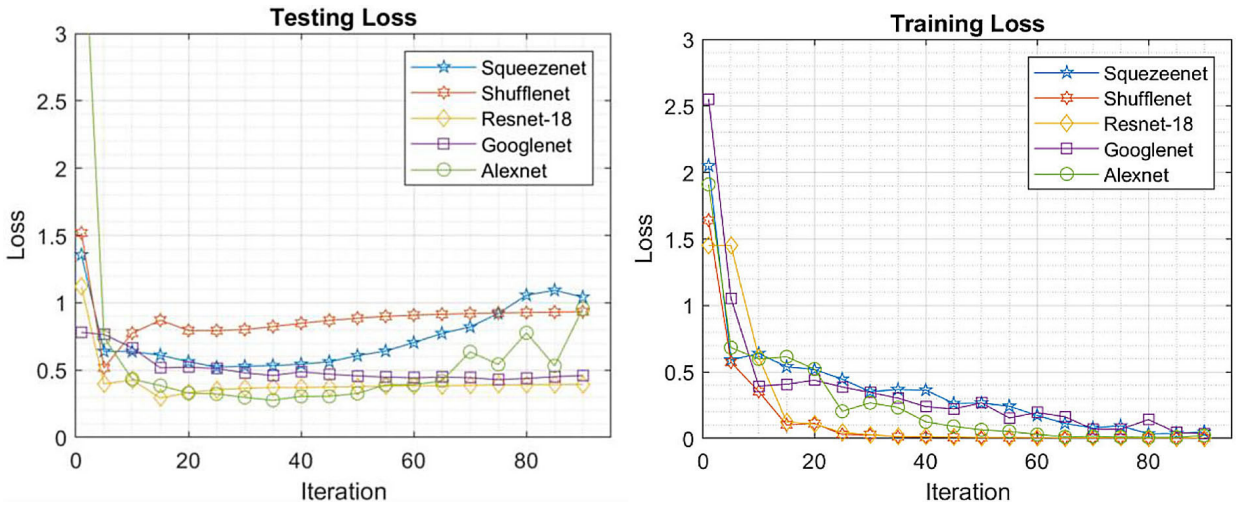

Fig. 4. Binary classification loss curves with Data 2 
also has the highest accuracy rate, this process can be provided from the loss curve in Figure 4. Among the loss curves in Figure 4, all curves are very close to zero and each other.

\section{CONCLUSIONS AND FUTURE DIRECTION}

In this study, a total of five classical pre-trained models were applied for the same purpose, with the help of the transfer learning approach, which has been previously used in many scientific studies and for many purposes. Therefore, five models were implemented to reach the target in the study. In this study, in order to interpret the scainer images and support to early diagnosis of the breast cancer, in addition to binary classification, quadruple classification was made. Two data sets were performed for this and five DCNN models were individually trained and tested with these two data sets. Since all models have applicability, the authors think that whatever model achieves the highest success is also the success of this research project. Because all DCNN models in this research project are designed and implemented for the same purpose. In this respect, the training and test results performed with computational tomography images can be evaluated separately for each data set.

In the applications made with the Data 2, approximately $86 \%$ accuracy rate was obtained. In other words, the diagnosis/prediction of the breast cancer and actionable tumor was made with accuracy rate of $86 \%$ with Resnet- 18 DCNN model. In the classification application with Data 1, the data set was made suitable for the quadruple classification. As a result of the testing, it was possible to accurately predict cancer with an accuracy rate of approximately $75 \%$. If it is necessary to make a general evaluation for the results of the this study, it is seen that the training accuracy rate and the test accuracy rates are not very close to each other especially for the quadruple classification. Conversely, for binary classification especially for Resnet-18, thee accuracy rate is very close to training rate. This indicates that the deep convolutional neural networks designed and implemented for this term are strong enough to diagnose and classify breast cancer based on image data.

In this study, the DL method was applied effectively. It can be observed that the pre-trained models are successful in terms of accuracy. Convolution and other hidden layers in the DCNN models are strong enough to automatically detect and classify the breast cancer CT image dataset. The DL method often requires a large amount of data for training. By using a sufficient number of image data, the reliability of the study was increased and using the fine tuning of the pre-trained DCNN models, it was possible to achieve about $86 \%$ of accuracy.

The system investigated in this research project is deep convolutional neural networks models that are performed for the diagnosis of breast cancer, based on image data only, without the need for any processing such as feature extraction from $\mathrm{CT}$ images.

\section{REFERENCES}

1. Abhinav G. Deep Learning Reading Group: SqueezeNet. KDnuggets; 2018.

2. Buda M., SahaA., Walsh R., Ghate S., Li N., Święcicki A., Lo J.Y., Yang J., Mazurowski M.A. Data from the Breast Cancer Screening - Digital Breast Tomosynthesis (BCS-DBT). Data from The Cancer Imaging Archive; 2020. DOI: 10.7937/e4wt-cd02.

3. Clark K., Vendt B., Smith K., Freymann J., Kirby J., Koppel P., Moore S., Phillips S., Maffitt D., Pringle M., Tarbox L., Prior F. The Cancer Imaging Archive (TCIA): Maintaining and Operating a Public Information Repository, Journal of Digital Imaging. 2013;26(6):1045-1057. DOI: 10.1007/ s10278-013-9622-7

4. Agnes S.A., Anitha J., Pandian S.I.A., Peter J.D. Classification of Mammogram Images Using Multiscale all Convolutional Neural Network (MACNN). Journal of Medical Systems. 2020;44(1):19. DOI: ARTN 30 10.1007/s10916-019-1494-Z

5. Berbar M.A. Hybrid methods for feature extraction for breast masses classification. Egyptian Informatics Journal. 2018;19(1):63-73. DOI:10.1016/j. eij.2017.08.001

6. Bharti D., Sahoo G.R., Shukla S., Pradhan A. Wavelet transform of Fourier domain low coherence interference spectra for breast cancer detection. Paper presented at the Tissue Optics and Photonics; 2020.

7. Binder A., Bockmayr M., Hagele M., Wienert S., Heim D., Hellweg K., Klauschen F. Morphological and molecular breast cancer profiling through explainable machine learning. Nature Machine Intelligence. 2021;3(4), 355-366. DOI:10.1038/ s42256-021-00303-4

8. Bishop C.M. Pattern Recognition and Machine Learning (Information Science and Statistics) (Softcover reprint of the original 1st ed. 2006 ed.). Springer. 2016.

9. Cuzick J., Sestak I., Forbes J.F., Dowsett M., Cawthorn S., Mansel R.E., Howell A.J.T.L. Use of an- 
astrozole for breast cancer prevention (IBIS-II): long-term results of a randomised controlled trial. 2020;395(10218):117-122.

10. De Santana, M.A., Pereira, J.M.S., da Silva W.W.A., dos Santos W.P. 2021. Breast Cancer Diagnosis in Mammograms Using Wavelet Analysis, Haralick Descriptors, and Autoencoder. In AI Innovation in Medical Imaging Diagnostics. IGI Global. 2021;76-91.

11. Desai M., Shah M.J.C.E. An anatomization on Breast Cancer Detection and Diagnosis employing Multi-layer Perceptron Neural Network (MLP) and Convolutional Neural Network (CNN); 2020.

12. Gupta K.K., Vijay R., Pahadiya P.J.S.C.T., Applications. A Review Paper on Feature Selection Techniques and Artificial Neural Networks Architectures Used in Thermography for Early Stage Detection of Breast Cancer. 2020;455-465.

13. Hakkoum H., Idri A., Abnane I. Assessing and comparing interpretability techniques for artificial neural networks breast cancer classication. Computer Methods in Biomechanics and Biomedical Engineering-Imaging and Visualization. 2021;113. DOI:10.1080/21681163.2021.1901784

14. He K, Zhang X., Ren S., Sun J. Deep Residual Learning for Image Recognition. IEEE Conference on Computer Vision and Pattern Recognition (CVPR), Las Vegas, NV, 2016, 770-778, DOI: 10.1109/CVPR.2016.90.

15. Hou C., Zhong X., He P., Xu B., Diao S., Yi F., Li J.J.J.M.I. Predicting breast cancer in Chinese women using machine learning techniques: algorithm development. 2020;8(6):e17364.

16. Kowal M., Filipczuk P., Obuchowicz A., Korbicz J., Monczak R. Computer-aided diagnosis of breast cancer based on fine needle biopsy microscopic images. Comput Biol Med. 2013;43(10):1563-1572. DOI: 10.1016/j.compbiomed.2013.08.003

17. Krizhevsky A., Sutskever I., Hinton G.E. ImageNet classification with deep convolutional neural networks. Communications of the ACM. 2017;.60(6):84-90. DOI:10.1145/3065386

18. Lei Y., He X., Yao J., Wang T., Wang L., Li W., Yang X.J.M.P. Breast tumor segmentation in 3D automatic breast ultrasound using Mask scoring RCNN. 2021;48(1):204-214.

19. Liu J., Cheng H., Lv X.Y., Zhang Z.X., Zheng X.X., Wu G.H., Yue X.X. Use of FT-IR spectroscopy combined with SVM as a screening tool to identify invasive ductal carcinoma in breast cancer. Optik. 2020;204:164225. DOI: ARTN 164225.10.1016/j. ijleo.2020.164225
20. Michelucci U. Advanced Applied Deep Learning: Convolutional Neural Networks and Object Detection (1st ed.). Apress; 2019.

21. Oh C. M., Lee D., Kong H.J., Lee S., Won Y.J., Jung K. W., Cho H. Causes of death among cancer patients in the era of cancer survivorship in Korea: Attention to the suicide and cardiovascular mortality. Cancer Medicine. 2020;9(5):1741-1752. DOI:10.1002/cam4.2813

22. Patnaik J.L., Byers T., DiGuiseppi C., Dabelea D., Denberg T.D.J.B.C.R. Cardiovascular disease competes with breast cancer as the leading cause of death for older females diagnosed with breast cancer: a retrospective cohort study. 2011;13(3):1-9.

23. Rasheed A., Younis M.S., Qadir J., Bilal M.J. a.p.a. Use of Transfer Learning and Wavelet Transform for Breast Cancer Detection; 2021.

24. Rawal G., Rawal R., Shah H., Patel K.. A Comparative Study Between Artificial Neural Networks and Conventional Classifiers for Predicting Diagnosis of Breast Cancer. In ICDSMLA 2019. Springer. 2020; 261-271.

25. Singh S. \& Kumar R. 2020. Histopathological image analysis for breast cancer detection using cubic SVM. Paper presented at the 2020 7th International Conference on Signal Processing and Integrated Networks (SPIN).

26. Szegedy C., Liu W., Jia Y., Sermanet P., Reed S., Anguelov D., Erhan D., Vanhoucke V., Rabinovich A. Going deeper with convolutions. IEEE Conference on Computer Vision and Pattern Recognition (CVPR) Boston MA USA 2015 June.

27. Vaka A.R., Soni B., Reddy S.J.I.E. Breast cancer detection by leveraging Machine Learning. 2020;6(4):320-324.

28. Vrigazova B.P. Detection of Malignant and Benign Breast Cancer Using the ANOVA-BOOTSTRAPSVM. Journal of Data and Information Science. 2020;5(2):62-75. DOI:10.2478/jdis-2020-0012

29. Yousefi H., Maheronnaghsh M., Molaei F., Mashouri L., Reza Aref A., Momeny M., Alahari S.K. Long noncoding RNAs and exosomal lncRNAs: classification, and mechanisms in breast cancer metastasis and drug resistance. Oncogene. 2020;39(5):953974. DOI: 10.1038/s41388-019-1040-y

30.Zhang M., Li,Q.J. a. p. a.. MS-GWNN: multiscale graph wavelet neural network for breast cancer diagnosis; 2020.

31. Zhang X., Zhou X., Lin M., Sun J. ShuffleNet: An Extremely Efficient Convolutional Neural Network for Mobile Devices. 2018 IEEE/CVF Conference on Computer Vision and Pattern Recognition, 2018, 6848-6856. DOI: 10.1109/CVPR.2018.00716 